\title{
Nanostructured Titanium Oxide Fabricated via Block Copolymer Template
}

\author{
Ryoko Watanabe, ${ }^{a}$ Tomokazu IYODA, ${ }^{\mathrm{a}}$ and Kaori ITO ${ }^{\mathrm{a}, \mathrm{b}}$ *
}

\begin{abstract}
aDivision of Integrated Molecular Engineering, Chemical Resources Laboratory, Tokyo Institute of Technology (R1254259 Nagatsuta-cho, Midori-ku, Yokohama, Kanagawa 226-8503, Japan)

bJST-PRESTO (4-1-8 Honmachi, Kawaguchi, Saitama 332-0012, Japan)
\end{abstract}

Received October 6, 2008 ; Accepted December 17, 2008

\begin{abstract}
Titanium tetrachloride vapor was selectively introduced into hexagonally arranged $\mathrm{PEO}$ cylinders in $\mathrm{PEO}_{\mathrm{m}}-b$ $\operatorname{PMA}(\mathrm{Az})_{\mathrm{n}}((\mathrm{m}, \mathrm{n})=(272,116),(272,94)$, and $(114,67))$ thin films by simply exposing the films to the vapor in a glass vessel. TEM images of $\mathrm{PEO}_{\mathrm{m}}-b$-PMA $(\mathrm{A} z)_{\mathrm{n}}$ thin films with $\mathrm{m}=272$ and 114 after the exposure showed hexagonally arranged dark spots with 14-nm diameter and 38-nm distance, and 10-nm diameter and 20-nm distance, respectively. FTIR measurement indicated a change in the peak shape at stretching vibration of C-O-C in the PEO chain as titanium tetrachloride was introduced. The film weight during the vapor process was increased in QCM measurement. XPS analysis was carried out for the films before and after hydrolysis with water vapor. It was found that titanium oxide with some different oxidation states existed in the hydrolysis treatment. FESEM revealed that calcination of the hydrolyzed film gave titanium oxide nanowires with about 10-nm diameter. XRD pattern of the calcined film suggested that the process demonstrated here generated the titanium oxide nanowires consisting of polymorph mixture with brookite, rutile, and anatase.
\end{abstract}

Key Words : Block Copolymer, PEO Cylinder, Nanotemplate, Titanium Oxide

\section{Introduction}

Nanostructures of lamellae, cylinders, spheres, and micelles offered by block copolymer (BC) self-assembly have been widely utilized as nanotemplates to fabricate nanostructured materials. The materials are inorganic semiconductor, metal, and metal oxide and also organic conductive polymers to reveal potential properties coming from nano-sized effects and their two or three dimensional periodic arrangements. Titanium oxide is one of the great target materials since it exhibits strong oxidizing properties and high refractive index to be applied to photocatalysts, ${ }^{1,2)}$ photovoltaic devices, ${ }^{3,4)}$ and optical materials. ${ }^{5)}$ While the effect of the crystallite size and shape of titanium oxide on the functions have been discussed, ${ }^{6-8)} \mathrm{BC}$ templateting processes have attracted much attention to fabricate the nanostructured titanium oxide. Thin films and micelle films of amphiphilic BCs, polystyrene- $b$-poly(ethylene oxide) (PS-b-PEO), ${ }^{9-14)}$ polystyrene- $b$-poly(2-vinyl-pyridine) (PS- $b$-P2VP), ${ }^{15,16)}$ polystyrene- $b$-poly (4-vinyl-pyridine) $\quad$ (PS- $b$-P 4 VP), ${ }^{17}$ ) poly(methylmethacrylate)-b-poly(ethylene oxide) (PMMA$b$-PEO), ${ }^{18,19)}$ and polyisoprene- $b$-poly(ethylene oxide) (PI- $b$ $\mathrm{PEO}{ }^{20)}$ have been used to selectively introduce titanium oxide precursor into a hydrophilic PEO, P2VP, and P4VP microdomains through solution and vapor processes. Titanium oxide nanodot arrays, ${ }^{9-11,13,15-17)}$ nanoplatelets, ${ }^{19)}$ nanobowls, ${ }^{11)}$ nanoporous structures, ${ }^{12,14,20)}$ and nanohybrid arrays ${ }^{18)}$ are fabricated to investigate the photochemical properties specific to the nanostructure. Anisotropic nanostructures of titanium oxide have been also demonstrated by multiple processes, in which the $\mathrm{BC}$ works as the first template then to transfer the nanostructures as the secondary templates to other materials. For example, needle-like titanium oxide grows from titanium nanoseeds, ${ }^{17)}$ and titanium oxide nanoposts are formed using sol-gel processes through nanopores of $\mathrm{BC}$ template. ${ }^{21)}$ Here, we demonstrate a simple preparative method for titanium oxide nanowires by applying titanium tetrachloride vapor as a precursor and PEO cylinder array as a template (Fig. 1). We have developed a liquid crystalline $\mathrm{BC}$ consisting of poly(ethylene oxide) and poly(methacrylate) bearing azobenzene mesogen in the side chain $\left(\mathrm{PEO}_{\mathrm{m}}-b\right.$ - $\left.\mathrm{PMA}(\mathrm{Az})_{\mathrm{n}}\right){ }^{22)}$ The chemical structure of $\mathrm{PEO}_{\mathrm{m}}-b-\mathrm{PMA}(\mathrm{Az})_{\mathrm{n}}$ is shown in Fig. 1. Thin films of $\mathrm{PEO}_{\mathrm{m}}-b-\mathrm{PMA}(\mathrm{Az})_{\mathrm{n}}$ exhibit hexagonally arranged $\mathrm{PEO}$ cylinders perpendicular to the film surface without any pretreatment of substrate surfaces. ${ }^{23-25)}$ The PEO cylinders have been used as nanotemplates to fabricate arrays of silica nanorods, ${ }^{26)}$ silver nanodots, ${ }^{27)}$ and leadhybridized PEO cylinders. ${ }^{28}$ PEO is a versatile medium for ions due to its ion-conductive and hydrophilic properties. In particular to our $\mathrm{BC}$, each $\mathrm{PEO}$ cylindrical

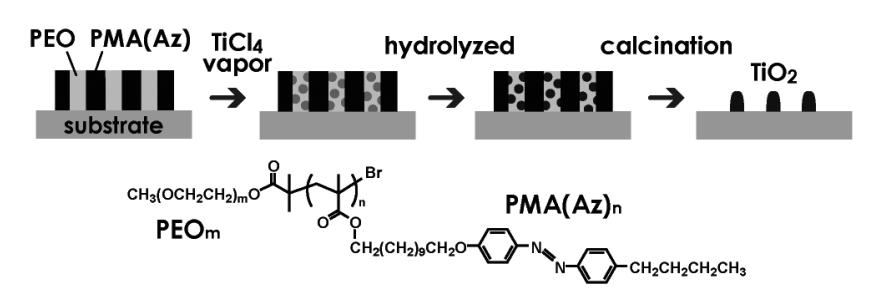

Fig. 1 Schematic illustration of the templating process using $\mathrm{PEO}_{\mathrm{m}}-b$-PMA $(\mathrm{Az})_{\mathrm{n}}$ block copolymer thin film and titanium tetrachloride $\left(\mathrm{TiCl}_{4}\right)$ vapor, and the chemical structure of $\mathrm{PEO}_{\mathrm{m}}-b-\mathrm{PMA}(\mathrm{Az})_{\mathrm{n}}((\mathrm{m}, \mathrm{n})=(272,116),(272,94)$, and $(114,67))$. 
microdomain in thin films is open on the outer surface, which offers easy incorporation of materials to be doped. We employed vapor processes desirable to keep the nanostructure of the $\mathrm{BC}$ nanotemplate because there is no physical damage on the thin films.

\section{Experimental}

Three BCs with different molecular weights were used as nanotemplates. The molecular weight of $\mathrm{PEO}_{272^{-}}$ $b$-PMA(Az) $)_{116}, \mathrm{PEO}_{272}-b$-PMA(Az) $)_{94}$, and $\mathrm{PEO}_{114}-b$ PMA(Az) 67 are $69.2 \mathrm{~kg} \mathrm{~mol}^{-1}$ (PEO: $12.1 \mathrm{~kg} \mathrm{~mol}^{-1}$, PMA(Az): $57.1 \mathrm{~kg} \mathrm{~mol}^{-1}$ ), $58.4 \mathrm{~kg} \mathrm{~mol}^{-1}$ (PEO: $12.1 \mathrm{~kg} \mathrm{~mol}^{-1}$, PMA(Az): $46.3 \mathrm{~kg} \mathrm{~mol}^{-1}$ ), and $38.1 \mathrm{~kg} \mathrm{~mol}^{-1}$ (PEO: $5.1 \mathrm{~kg}$ $\mathrm{mol}^{-1}$, PMA(Az): $33.0 \mathrm{~kg} \mathrm{~mol}^{-1}$ ), respectively. They were synthesized according to our previous report.22) Titanium tetrachloride was used as received from Wako Pure Chemical Industries, Ltd. BC thin films as nanotemplates were prepared by spin-coating $2.5 \mathrm{wt} \%$ toluene solutions of each $\mathrm{BC}$ onto silicon wafers. For transmission electron microscopic (TEM) observation, the toluene solution was spread on a water surface. Thin films formed on the water surface were transferred onto cupper grids. Thermal annealing at $140{ }^{\circ} \mathrm{C}$, above its isotropic transition at around $120^{\circ} \mathrm{C}$, for $2 \mathrm{~h}$ under vacuum was performed to induce the hexagonally arranged PEO cylinders. As a doping process of titanium oxide precursors, $\mathrm{BC}$ films were exposed to the vapor in a closed glass vessel $(150 \mathrm{ml})$ containing $5 \mathrm{ml}$ of titanium tetrachloride under a cool condition at around $0{ }^{\circ} \mathrm{C}$ provided by an ice bath. TEM observation was performed with an H-7000 (Hitachi High-Technologies) at an accelerating voltage of $100 \mathrm{kV}$. Fourier transform infrared spectroscopy (FTIR) was carried out with an FT/IR-660 plus (JASCO). A setup for the quarts crystal microbalance (QCM) measurement was assembled with an HQ-601DK (mass sensor), an HX-Q1 $=10 \mathrm{M}$ (quartz crystal oscillator with a polished gold electrode, $10 \mathrm{MHz}$ ), and an HQ-101B (controller) manufactured by Hokuto Denko Corp. Quartz crystal oscillators were used as received. BC solutions were directly spin-coated on the quartz crystal oscillators followed by thermal annealing as described above. Exposure of titanium tetrachloride vapor was performed using a cooled vessel $(100 \mathrm{ml})$ with a shutter between the sample and the vapor. X-ray photoelectron spectroscopy (XPS) was carried out with an ESCA-3400 (SHIMADZU Corp.) with $\mathrm{Mg} \mathrm{K} \alpha$ excitation at $300 \mathrm{~W}$. Field emission scanning electron microscope (FESEM) image was recorded using an S-5200 (Hitachi HighTechnologies). X-ray diffraction (XRD) patterns were collected by an X-ray diffractometer (Rigaku, RintUltimaIII) with $\mathrm{Cu} K \alpha$ radiation $(\lambda=0.15418 \mathrm{~nm})$ source at $40 \mathrm{kV}$ and $20 \mathrm{~mA}$.

\section{Results and Discussion}

Figures 2a and 2c show TEM images of $\mathrm{PEO}_{272}-b$ $\operatorname{PMA}(\mathrm{Az})_{94}$ and $\mathrm{PEO}_{114}-b$-PMA(Az) $)_{67}$ thin films, respectively, stained with ruthenium oxide to visualize the microphase-separated structure. In the both films, selectively stained PEO cylinders were imaged as hexagonally arranged dark dots. The average diameter $(d)$ and

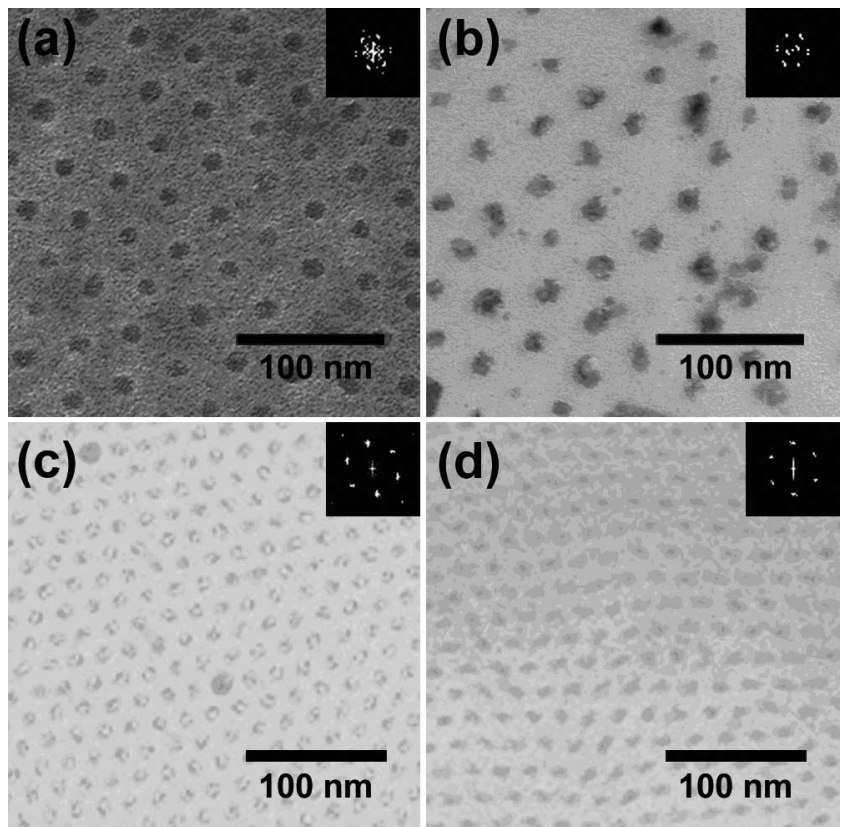

Fig. 2 TEM images of $\mathrm{PEO}_{272}-b$-PMA $(\mathrm{Az})_{94}(\mathrm{a}, \mathrm{b})$ and $\mathrm{PEO}_{114}-b$-PMA $(\mathrm{Az})_{67}$ (c, d) thin films with different pretreatment for the observations: $(\mathrm{a}, \mathrm{c})$ ruthenium staining, (b, d) titanium tetrachloride vapor doping for $5 \mathrm{~min}$. The FFT images are shown in insets.

center-to-center distance between adjacent PEO cylinders $(D)$ were estimated to be $d=15 \mathrm{~nm}$ and $D=40 \mathrm{~nm}$ for $\mathrm{PEO}_{272}-b-\mathrm{PMA}(\mathrm{Az})_{94}$, and $d=10 \mathrm{~nm}$ and $D=20 \mathrm{~nm}$ for $\mathrm{PEO}_{114}-b-\mathrm{PMA}(\mathrm{Az})_{67}$, reflecting from the molecular weight of each segment. The $\mathrm{BC}$ thin films exposed to titanium tetrachloride vapor for 5 min (Figs. $2 \mathrm{~b}$ and $2 \mathrm{~d}$ ) exhibited the hexagonally arranged dark spots with reasonably consistent $d$ and $D\left(\mathrm{PEO}_{272}-b \text {-PMA(Az) }\right)_{94}: d=14$ nm, $D=38 \mathrm{~nm}, \mathrm{PEO}_{114}-b-\mathrm{PMA}(\mathrm{Az})_{67}: d=10 \mathrm{~nm}, D=21$ $\mathrm{nm}$ ) with those of the $\mathrm{BC}$ films stained with ruthenium oxide. Since a dark contrast in TEM images is attributed to presence of heavy elements in a sample, these films can be assigned that PEO cylinders were selectively doped with titanium tetrachloride and its hydrolyzed product. The PEO-selective diffusion of doping sources was originated from chemical interactions such as the hydrogen and coordination bondings with the ethylene oxide units. ${ }^{28)}$ In the present vapor process with titanium tetrachloride, $\mathrm{Ti}^{4+}$, $\mathrm{Ti}(\mathrm{OH})_{2}{ }^{2+}$, and $\mathrm{Ti}(\mathrm{OH})_{4}$ formed with the humidity were supposed to penetrate into the PEO cylinders through ionic-polar interactions. ${ }^{6,17,29)}$ FTIR spectra of $\mathrm{PEO}_{114}-b$-PMA(Az) $)_{67}$ thin films with different doping times $(0,1,5$, and $10 \mathrm{~min})$ showed gradual changes in stretching vibration peaks of C-O-C of PEO at $1107 \mathrm{~cm}^{-1}, 1144 \mathrm{~cm}^{-1}$, and $1150 \mathrm{~cm}^{-1}$ (Fig. 3). The intensity of peak at $1107 \mathrm{~cm}^{-1}$ decreased and $1144 \mathrm{~cm}^{-1}$ and $1150 \mathrm{~cm}^{-1}$ increased as the doping progressed. The peaks in the $\mathrm{C}-\mathrm{O}-\mathrm{C}$ stretching region are so sensitive to the surrounding conditions. The changes of peak intensities in the current system were in good agreement with those in existence of chemical interactions between cations and oxygens in oligoether, indicating the appreciable interactions of the cations and $\mathrm{PEO}{ }^{30,31)} \mathrm{A}$ part of the 


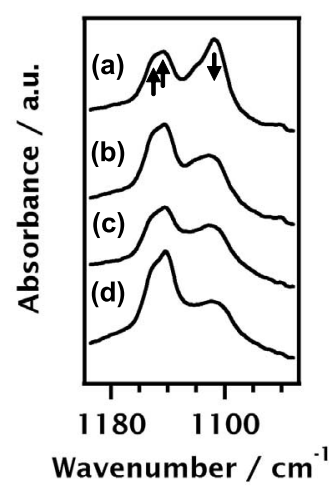

Fig. 3 FTIR spectra of C-O-C stretching vibration modes in the $\mathrm{PEO}_{114}-b$-PMA $(\mathrm{Az})_{67}$ thin films exposed to titanium tetrachloride vapor for $0 \mathrm{~min}(\mathrm{a}), 1 \mathrm{~min}$ (b), $5 \mathrm{~min}$ (c), and 10 $\min (\mathrm{d})$.

resulting titanium oxide would give the particles under the present condition, which are not crystal structures without any further treatment such as thermal annealing. ${ }^{629)}$ The penetration of titanium compounds were possibly confirmed by cross-sectional TEM images illustrating wire structures inside of the film. In our further study, the preparation of the section specimen to obtain fine TEM images should be improved for the detailed discussions.

The doping process was monitored by QCM to orderquantitatively estimate the amount of titanium compound doped into PEO cylinders. Figure 4 shows a profile obtained when the exposure of titanium tetrachloride vapor for $30 \mathrm{sec}$ was repeated three times. When the shutter was opened (indicated by $\mathrm{x}$ in Fig. 4), the frequency intensively decreased, implying the episodic adsorption of the vapor. The frequency recovered to be constant within 20 min once the shutter was closed. We focused on the difference of frequency between the point A $(-59.6 \mathrm{~Hz})$ and B $(-93.9 \mathrm{~Hz})$ in Fig 4, meaning the frequency change for 90 -sec (30 sec $\times 3$ times) doping. Since the quartz crystal oscillator has a characteristic as 2.95 $\mathrm{Hz} / \mathrm{ng}$, the frequency change was converted to weight gain with $12 \mathrm{ng}$, which indicates the incorporation of the vapor into the $\mathrm{BC}$ film coated on the oscillator. The result in weight gain (12 $\mathrm{ng})$ can be roughly estimated that $20 \mathrm{vol} \%$ of one PEO cylinder, e.g., $d=7 \mathrm{~nm}$ and $D=$ $20 \mathrm{~nm}$, was occupied with titanium tetrachloride, under the assumption that the species doped was only titanium tetrachloride and the thickness of the $\mathrm{BC}$ film on the oscillator was $100 \mathrm{~nm}$. We found the considerable mass change with the QCM. It should be noted that QCM has a potential to evaluate mass changes during the vapor doping process into $\mathrm{BC}$ templates. Independent analysis such as ICP will calibrate the estimate by QCM.

Fabrication of titanium oxide nanowires was demonstrated by calcinating the $\mathrm{BC}$ template after the doping process. As the Step 1, $\mathrm{PEO}_{272}-b$-PMA(Az) $)_{116}$ thin film was exposed to titanium tetrachloride vapor for $5 \mathrm{~min}$. The sample was then placed in a closed vessel containing water for $2 \mathrm{~h}$ at $50^{\circ} \mathrm{C}$ to completely hydrolyze the remaining titanium tetrachloride in PEO cylinders (Step

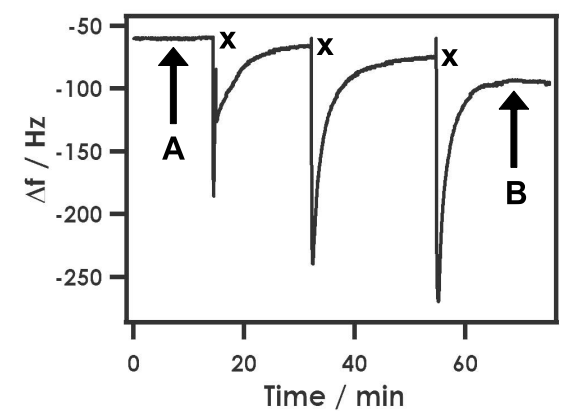

Fig. 4 QCM profile in the titanium tetrachloride doping process using spin-coated film of $\mathrm{PEO}_{114}-b-\mathrm{PMA}(\mathrm{Az})_{67}$. The shutter was opened to expose the vapor for $30 \mathrm{sec}$ at the point indicated by $\mathrm{x}$ in the figure.
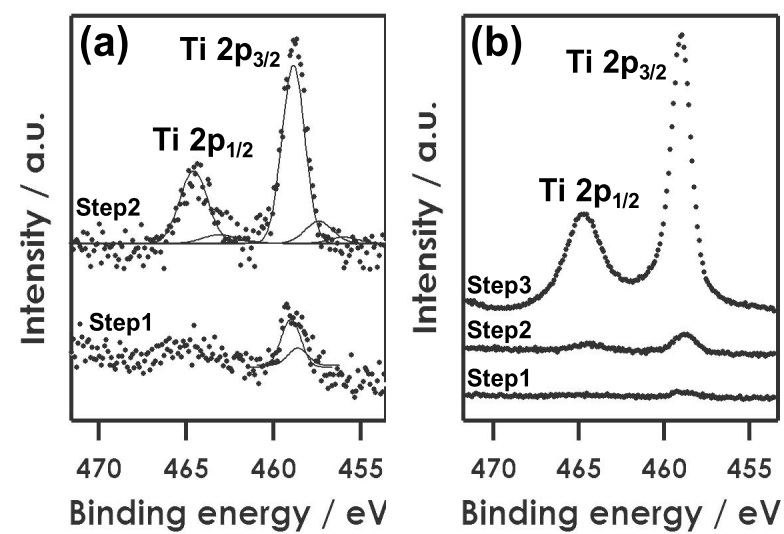

Fig. 5 XPS Ti $2 p$ spectra for $\mathrm{PEO}_{272}-b$-PMA(Az) ${ }_{116}$ thin films doped with titanium tetrachloride for $5 \mathrm{~min}$. (a) As doped (Step 1) and hydrolyzed film in a closed vessel containing water for $2 \mathrm{~h}$ at $50{ }^{\circ} \mathrm{C}$ (Step 2). (b) The film calcined at $550{ }^{\circ} \mathrm{C}$ for $1 \mathrm{~h}$ (Step 3). The peak intensities of the sample at the Step 1 and 2 were quite weaker than that in Step 3.

2). Finally, the sample was calcined at $550^{\circ} \mathrm{C}$ for $1 \mathrm{~h}$ to crystallize the titanium species and remove the $\mathrm{BC}$ template (Step 3). Figure 5a shows XPS Ti $2 p$ spectra of the samples at the Step1 and Step 2. The Step 1 gave a main peak at $459.0 \mathrm{eV}$ and at $458.3 \mathrm{eV}$ with very weak intensity. The peak at the lower energy could be attributed to $\mathrm{Ti}^{4+}$ species. ${ }^{32)}$ Hydrolysis (Step 2) gave a doublet peak attributable to titanium dioxide $\left(\mathrm{TiO}_{2}\right)$ at $458.9 \mathrm{eV}\left(2 \mathrm{p}_{3 / 2}\right)$ and $464.6 \mathrm{eV}\left(2 \mathrm{p}_{1 / 2}\right)$ including other oxidation states such as $\mathrm{Ti}_{2} \mathrm{O}_{3}\left(457.4 \mathrm{eV}\right.$ for $2 \mathrm{p}_{3 / 2}$ and $463.1 \mathrm{eV}$ for $\left.2 \mathrm{p}_{1 / 2}\right)$ and TiO (456.0 eV for $2 \mathrm{p}_{3 / 2}$ and $461.7 \mathrm{eV}$ for $\left.2 \mathrm{p}_{1 / 2}\right)^{18,33,34)}$ After calcination (Step 3), due to the absence of the BC template, the peaks of titanium oxide became intense (Fig. $5 b)$. The spectrum at Step 3 indicates a doublet at 459.04 $\mathrm{eV}\left(2 \mathrm{p}_{3 / 2}\right)$ and $464.74 \mathrm{eV}\left(2 \mathrm{p}_{1 / 2}\right)$. The difference between the doublet peaks is $5.7 \mathrm{eV}$ and the area ratio of the two peaks $\left(2 \mathrm{p}_{3 / 2} / 2 \mathrm{p}_{1 / 2}\right)$ is 0.51 , which fairly agree with the oxidation state of $\mathrm{TiO}_{2}{ }^{18,33,34)}$ The XPS characterizations ensured us that titanium oxide precursor was adequately incorporated in the $\mathrm{BC}$ film and titanium dioxide was mainly formed through the hydrolysis and the calcination. The fabrication process resulted in removal of the 
$\mathrm{BC}$ template and gave more homogenous titanium oxide. For FESEM observation, cast film of $\mathrm{PEO}_{272}-b$ $\operatorname{PMA}(\mathrm{Az})_{116}$ template (concentration of the cast solution: $2.5 \mathrm{wt} \%$, thickness: a few micrometers) on silicon wafer was doped with titanium tetrachloride for $10 \mathrm{~min}$. Hydrolysis $\left(50^{\circ} \mathrm{C}, 2 \mathrm{~h}\right)$ and calcination $\left(550^{\circ} \mathrm{C}, 1 \mathrm{~h}\right)$ provided titanium oxide nanowires, which appear as randomly dispersed bright contrast lines on the silicon wafer in the FESEM image (Fig. 6). The diameter of the nanowire was about $10 \mathrm{~nm}$, while the diameter of the PEO cylinder in the $\mathrm{BC}$ template was about $15 \mathrm{~nm}$. When assumed the $\mathrm{BC}$ template shrunk by calcination, it is reasonable that the diameter of the resulting nanowire became smaller. The length of the nanowires reached up to several hundred nanometers, which could be controlled by the thickness of the $\mathrm{BC}$ template. Although we need to optimize the conditions of the calcination process to keep the ordered arrangement and perpendicular orientation of the PEO cylinder arrays in the BC template, the demonstration to fabricate nanowires of titanium oxide by removing $\mathrm{BC}$ template was succeeded through the simple vapor doping process.

Crystal phase of titanium oxide nanowires fabricated through the present $\mathrm{BC}$ template method was investigated by XRD. However, no diffraction peaks were obtained from the resulting titanium oxide nanowires shown in Fig. 6. Since the nanowires formed only monolayer structure on the silicon wafer, the amount was too small to give significant diffraction peaks. Yuwono et al. also reported that the nanohybrid thin film of PMMA-b-PEO with titania nanoparticle (thickness: less than $50 \mathrm{~nm}$ ) did not show any noticeable peaks in XRD measurement, implying the difficulty of the phase identification of nanostructured materials. ${ }^{18)}$ To overcome this problem, thicker cast film was prepared with $5 \mathrm{wt} \%$ toluene solution of $\mathrm{PEO}_{272}-b$-PMA(Az) $)_{116}$ on glass substrate $(76 \mathrm{~mm} \times$ $26 \mathrm{~mm}$ ) and 30-min titanium tetrachloride doping was performed. This preparation resulted in relatively large amount of titanium oxide nanowires forming network nanostructures over the entire surface of the glass substrate. The titanium oxide nanowires were then scratched off from the glass substrate and the resultant powder specimen of titanium oxide nanowire aggregates was finally obtained for XRD measurement. The XRD pattern (Fig. 7) with peaks at $2 \theta=28.3,32.7,47.2$, and $56.3^{\circ}$ was obtained, which suggests the titanium oxide nanowires exhibit polymorphic crystals composed of three different phases of brookite, rutile, and anatase. Although the peak intensities are relatively weak due to the small amount of the specimen, the peak positions are in reasonable agreements with the literatures, ${ }^{35-37)}$ in which the phase content in different kinds of polymorphs of titanium oxides are discussed. It is also known that the high acidity generated by titanium tetrachloride prefers the formation of brookite phase. ${ }^{35,36)}$ Thus, the brookite is supposed to be a major component. The crystallite size was estimated to be around $12 \mathrm{~nm}$ from Scherrer equation, in which Scherrer constant was 0.9 and the half width of the diffraction peaks was obtained after gauss fitting. ${ }^{38)}$ As compared with the width of

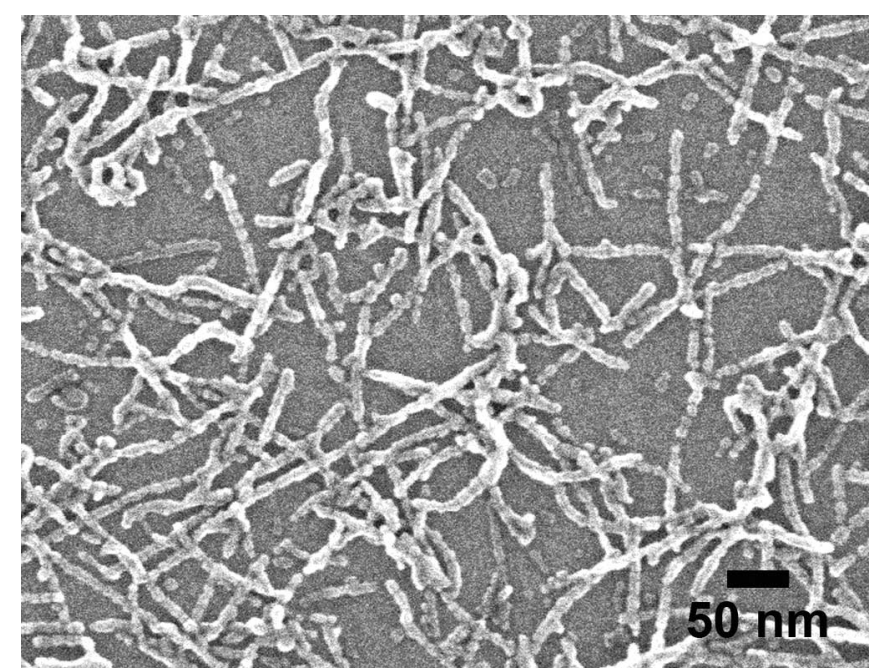

Fig. 6 FESEM image of titanium oxide nanowires fabricated through titanium tetrachloride doping (10 min), hydrolysis $\left(50^{\circ} \mathrm{C}, 2 \mathrm{~h}\right)$, and calcination $\left(550{ }^{\circ} \mathrm{C}, 1 \mathrm{~h}\right)$ by using a cast film of $\mathrm{PEO}_{272}-\mathrm{b}-\mathrm{PMA}(\mathrm{Az})_{116}$ as the template. The width of nanowires is about $10 \mathrm{~nm}$.

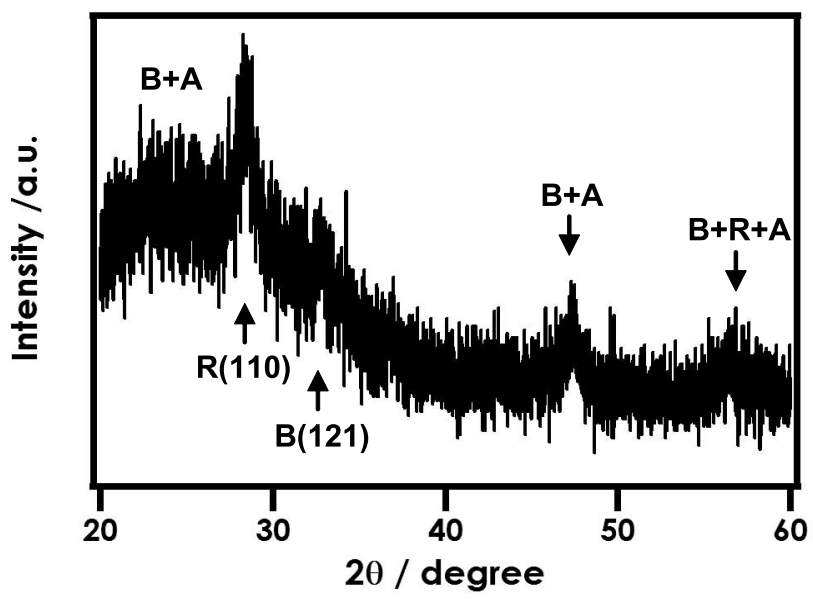

Fig. 7 XRD pattern obtained from aggregates of titanium oxide nanowires scratched off from the glass substrate. The nanowires were fabricated through titanium tetrachloride doping (30 min), hydrolysis $\left(50^{\circ} \mathrm{C}, 2 \mathrm{~h}\right)$ and calcination $\left(550{ }^{\circ} \mathrm{C}, 1 \mathrm{~h}\right)$ by using a thick cast film of $\mathrm{PEO}_{272}-b$ $\operatorname{PMA}(\mathrm{Az})_{116}$ on a glass substrate as the template. The XRD pattern corresponds to a titanium oxide polymorph consisting of brookite (B), rutile (R), and anatase (A).

nanowires estimated from the FESEM image (Fig. 6), the crystallite size given by Scherrer equation is reasonable. High resolution TEM observation is now undergoing to adequately reveal the crystal phase and size.

\section{Conclusion}

The $\mathrm{PEO}_{\mathrm{m}}-b$-PMA $(\mathrm{Az})_{\mathrm{n}}$ thin films with perpendicular PEO cylinders were applied as nanotemplates to fabricate titanium oxide nanowires by the simple templating process using titanium tetrachloride vapor as a precursor. The nanowires with 10 -nm diameter and severalhundred $\mathrm{nm}$ length were revealed to have polymorphic crystalline structures of titanium oxide. This vapor 
process takes advantages of the ion-conductive and hydrophilic properties of the PEO cylindrical microdomain as well as their perpendicular alignment, opening up the fabrication method for a variety of nanomaterials. The nanowire structure of titanium oxide, which has high refractive index, could be applied for a new optical filter with gradient refractive index and other optical devices. As we are able to tune the diameter and distance of PEO cylinders independently by changing molecular weight of the each segment, ${ }^{25)}$ effects of the feature size and the periodicity on the photocatalytic properties could be investigated in future.

\section{Acknowledgement}

This work was supported by the Foundation for the Promotion of Industrial Science, JST (PRESTO) and JSPS (Grant-in-Aid for Young Scientists (A)). We would like to thank Sumitomo Chemical Co., Ltd. for the financial support. We are grateful to Dr. Toshiyuki Yokoi for his meaningful discussion on the measurement of XRD and to Prof. Takashi Tatsumi for the use of FESEM.

\section{References}

1) A. Fujishima and K. Honda, Nature, 238, 37 (1972).

2) A. L. Linsebigler, G. Lu, and J. T. Yates, Jr., Chem. Rev., 95, 735 (1995).

3) U. Bach, D. Lupo, P. Comte, J. E. Moser, F. Weissörtel, J. Salbeck, H. Spreitzer, and M. Grätzel, Nature, 395, 583 (1998).

4) M. Zukalová, A. Zukal, L. Kavan, M. K. Nazeeruddin, P. Liska, and M. Grätzel, Nano Letters, 5, 1789 (2005).

5) S. Jeon and P. V. Braun, Chem. Mater., 15, 1256 (2003).

6) N. Serpone, D. Lawless, and R. Khairutdinov, J. Phys. Chem., 99, 16646 (1995).

7) Y. Sakatani, D. Grosso, L. Nicole, C. Boissière, G. J. d. A. A. Soler-Illia, and C. Sanchez, J. Mater. Chem., 16, 77 (2006).

8) D. S. Kim, S. J. Han, and S.-Y. Kwak, J. Colloid Interface Sci., 316, 85 (2007).

9) J. Gutierrez, A. Tercjak, I. Garcia, L. Peponi, and I. Mondragon, Nanotechnology, 19, 155607 (2008).

10) D. H. Kim, S. H. Kim, K. Lavery, and T. P. Russell, Nano Letters, 4, 1841 (2004).

11) J. Peng, X. Li, D. H. Kim, and W. Knoll, Macromol. Rapid Commun., 28, 2055 (2007).

12) B. Ma, J. Ma, and G. K. L. Goh, J. Mater. Sci., 43, 4297 (2008).

13) Z. Sun, D. H. Kim, M. Wolkenhauer, G. G. Bumbu, W. Knoll, and J. S. Gutmann, Chem. Phys. Chem., 7, 370 (2006).

14) O.-H. Park, J. Y. Cheng, H. S. Kim, P. M. Rice, T. Topuria, R. D. Miller, and H.-C. Kim, Appl. Phys. Lett., 90,
233102 (2007).

15) X. Li, K. H. A. Lau, D. H. Kim, and W. Knoll, Langmuir, 21, 5212 (2005).

16) J. Peng, W. Knoll, C. Park, and D. H. Kim, Chem. Mater., 20, 1200 (2008).

17) C.-C. Weng, K.-F. Hsu, and K.-H. Wei, Chem. Mater., 16, 4080 (2004).

18) A. H. Yuwono, Y. Zhang, J. Wang, X. H. Zhang, H. Fan, and W. Ji, Chem. Mater., 18, 5876 (2006).

19) Y. Cheng, M. Memesa, S. Nett, and J. S. Gutmann, Mater. Res. Soc. Symp. Proc., 974E, No pp given (2007).

20) J. Lee, M. C. Orilall, S. C. Warren, M. Kamperman, F. J. DiSalvo, and U. Wiesner, Nature Materials, 7, 222 (2008).

21) O.-H. Park, J. Y. Cheng, M. W. Hart, T. Topuria, P. M. Rice, L. E. Krupp, R. D. Miller, H. Ito, and H.-C. Kim, Adv. Mater., 20, 738 (2008).

22) Y. Tian, K. Watanabe, X. Kong, J. Abe, and T. Iyoda, Macromolecules, 35, 3739 (2002).

23) K. Watanabe, H. Yoshida, K. Kamata, and T. Iyoda, Trans. Mater. Soc. Jpn., 30, 377 (2005).

24) M. Komura and T. Iyoda, Macromolecules, 40, 4106 (2007).

25) R. Watanabe, K. Kamata, and T. Iyoda, J. Mater. Chem., 18, 5482 (2008).

26) A. Chen, M. Komura, K. Kamata, and T. Iyoda, Adv. Mater., 20, 763 (2007).

27) J. Li, K. Kamata, S. Watanabe, and T. Iyoda, Adv. Mater., 19, 1267 (2007).

28) S. Suzuki, K. Kamata, H. Yamauchi, and T. Iyoda, Chem. Lett., 36, 978 (2007).

29) Q. Zhang, L. Gao, and H. Xie, Mater. Sci. Eng., A, 343, 22 (2003).

30) S. J. Wen, T. J. Richardson, D. I. Ghantous, K. A. Striebel, P. N. Ross, and E. J. Cairns, J. Electroanal. Chem., 408, 113 (1996).

31) J. Li, K. Kamata, M. Komura, T. Yamada, H. Yoshida, and T. Iyoda, Macromolecules, 40, 8125 (2007).

32) H. Tiznado and F. Zaera, J. Phys. Chem. B, 110, 13491 (2006).

33) J. Pouilleau, D. Devilliers, H. Groult, and P. Marcus, J. Mater. Sci., 32, 5645 (1997).

34) P. M. Kumar, S. Badrinarayanan, and M. Sastry, Thin Solid Films, 358, 122 (2000).

35) A. Pottier, C. Chanéac, E. Tronc, L. Mazerolles, and J. P. Jolivet, J. Mater. Chem., 11, 1116 (2001).

36) S. Bakardjieva, V. Stengl, L. Szatmary, J. Subrt, J. Lukac, N. Murafa, D. Niznansky, K. Cizek, J. Jirkovskyc, and N. Petrova, J. Mater. Chem., 16, 1709 (2006).

37) Y. Zhang, L. Wu, Q. Zeng, and J. Zhi, J. Phys. Chem. C, 112, 16457 (2008).

38) S. Kitazawa, Y. Choi, S. Yamamoto, and T. Yamaki, Thin Solid Films, 515, 1901 (2006). 